\title{
Errata: Adaptive remote sensing image fusion under the framework of data assimilation
}

\author{
Rongyuan Chen \\ Hunan University of Commerce \\ Department of Information \\ Changsha, 410205, China \\ and \\ Wuhan University \\ State Key Laboratory of Information Engineering in \\ Surveying, Mapping and Remote Sensing \\ Wuhan, 430079, China
}

\section{Wei Xie}

Wuhan University

Computer School

129 Luoyu Road

Wuhan, 430079, China

E-mail: wxie.imageprocessing@gmail.com

\section{Leiguang Wang \\ Qianqing Qin}

Wuhan University

State Key Laboratory of Information Engineering in Surveying, Mapping and Remote Sensing

Wuhan, 430079, China

[DOI: $10.1117 / 1.3600346]$

This article [Opt. Eng. 50, 067006 (2011)] was originally published on 20 May 2011 with an incorrect citation identifier (CID).

The typical structure of the six-digit CID for OE uses the first two digits to indicate the volume number, and the middle two digits to indicate the section category. This paper was originally published under the heading "Special Section on Infrared Detectors" (section code 10), but has been republished as a regular paper under the category "Image Processing," which uses section code 70. The updated CID is 067006.

The manuscript was corrected online on 25 May 2011. The article appears correctly in print.

For more information on the use of CIDs in OE, see http://spiedigitallibrary.org/oe/citation_format. 